\title{
UNIFORM CONTROLLABILITY OF THE LINEAR ONE DIMENSIONAL SCHRÖDINGER EQUATION WITH VANISHING VISCOSITY
}

\author{
SORIN Micu ${ }^{1}$ AND IONEL ROVEnŢA ${ }^{1}$
}

\begin{abstract}
This article considers the linear 1-d Schrödinger equation in $(0, \pi)$ perturbed by a vanishing viscosity term depending on a small parameter $\varepsilon>0$. We study the boundary controllability properties of this perturbed equation and the behavior of its boundary controls $v_{\varepsilon}$ as $\varepsilon$ goes to zero. It is shown that, for any time $T$ sufficiently large but independent of $\varepsilon$ and for each initial datum in $H^{-1}(0, \pi)$, there exists a uniformly bounded family of controls $\left(v_{\varepsilon}\right)_{\varepsilon}$ in $L^{2}(0, T)$ acting on the extremity $x=\pi$. Any weak limit of this family is a control for the Schrödinger equation.
\end{abstract}

Mathematics Subject Classification. 93B05, 30E05, 35Q41.

Received July 14, 2010. Revised October 25, 2010.

Published online January 19, 2011.

\section{INTRODUCTION}

Given a time $T>0$ and an initial datum $y^{0} \in H^{-1}(0, \pi)$, the null-controllability property of the linear 1-d Schrödinger equation

$$
\left\{\begin{array}{l}
y_{t}(t, x)-\mathrm{i} y_{x x}(t, x)=0, \quad x \in(0, \pi), t>0 \\
y(t, 0)=0, y(t, \pi)=v(t), \quad t>0 \\
y(0, x)=y^{0}(x), \quad x \in(0, \pi)
\end{array}\right.
$$

consists of finding a scalar function $v \in L^{2}(0, T)$, called control, such that the corresponding solution $y$ of $(1.1)$ verifies $y(T, \cdot)=0$. There exists a huge literature concerning the controllability of both linear and nonlinear Schrödinger equation. We refer the interested reader to the pioneering articles [20,23], the monographs $[6,30]$ and the references therein. A great variety of techniques have been applied in these studies such as the Hilbert Uniqueness Method, multipliers, Carleman inequalities and so on. In the one dimensional case (1.1), a control $v$ may be constructed by means of a biorthogonal family to the sequence of exponential functions $\left(\mathrm{e}^{\nu_{n} t}\right)_{n \geq 1}$, where $\nu_{n}=\mathrm{i} n^{2}$ are the eigenvalues of the unbounded skew-adjoint differential operator corresponding to (1.1). We recall that a family of functions $\left(\phi_{m}\right)_{m \geq 1} \subset L^{2}\left(-\frac{T}{2}, \frac{T}{2}\right)$ with the property that

$$
\int_{-\frac{T}{2}}^{\frac{T}{2}} \phi_{m}(t) \mathrm{e}^{\bar{\nu}_{n} t} \mathrm{~d} t=\delta_{m n} \quad \forall m, n \geq 1
$$

\footnotetext{
Keywords and phrases. Null-controllability, Schrödinger equation, complex Ginzburg-Landau equation, moment problem, biorthogonal, vanishing viscosity.

${ }^{1}$ Facultatea de Matematica si Informatica, Universitatea din Craiova, 200585 Craiova, Romania. sd_micu@yahoo.com; roventaionel@yahoo.com
} 
is called a biorthogonal sequence to $\left(\mathrm{e}^{\nu_{n} t}\right)_{n \geq 1}$ in $L^{2}\left(-\frac{T}{2}, \frac{T}{2}\right)$. In (1.2), $\delta_{m n}$ stands for the Kronecker symbol which is 1 if $n=m$ and 0 otherwise.

In the particular case $\nu_{n}=\mathrm{i} n^{2}$, the existence of a biorthogonal sequence to $\left(\mathrm{e}^{\nu_{n} t}\right)_{n \geq 1}$ is a consequence of Ingham's inequality

$$
\sum_{n \geq 1}\left|a_{n}\right|^{2} \leq C(T) \int_{-\frac{T}{2}}^{\frac{T}{2}}\left|\sum_{n \geq 1} a_{n} \mathrm{e}^{\nu_{n} t}\right|^{2} \mathrm{~d} t \quad \forall\left(a_{n}\right)_{n \geq 1} \in \ell^{2},
$$

which holds for any $T>0$ due to the fact that $\lim \inf _{n \rightarrow \infty}\left|\nu_{n+1}-\nu_{n}\right|=\infty$ (see $\left.[3,16]\right)$.

Once a family of functions $\left(\phi_{m}\right)_{m \geq 1}$ verifying (1.2) is given, a control $v(t)$ for (1.1) is obtained by considering linear combinations of them. More precisely, if $y^{0}=\sum_{n \geq 1} a_{n} \sin (n x)$, then

$$
v(t)=\sum_{n \geq 1} \frac{(-1)^{n} \pi a_{n}}{2 n \mathrm{i}} \phi_{n}\left(t-\frac{T}{2}\right) \mathrm{e}^{-\frac{T}{2} \bar{\nu}_{n}} \quad \forall t \in(0, T)
$$

is a control which leads the solution $y$ of (1.1) to zero in time $T$, provided that the series converges in $L^{2}(0, T)$.

In this article, we study the possibility of obtaining a control for (1.1) as limit of controls of the following perturbed equations

$$
\left\{\begin{array}{l}
y_{t}(t, x)-\mathrm{i} y_{x x}(t, x)-\varepsilon y_{x x}(t, x)=0, \quad x \in(0, \pi), t>0 \\
y(t, 0)=0, y(t, \pi)=v_{\varepsilon}(t), \quad t>0 \\
y(0, x)=y^{0}(x), \quad x \in(0, \pi)
\end{array}\right.
$$

where $\varepsilon$ is a small parameter devoted to tend to zero. A function $v_{\varepsilon} \in L^{2}(0, T)$ is called control for the initial datum $y^{0}$ in time $T$ if the corresponding solution of (1.5) verifies $y(T, \cdot)=0$. If, given any $y^{0} \in H^{-1}(0, \pi)$, there exists a control $v_{\varepsilon} \in L^{2}(0, T)$ for $y^{0}$ in time $T$, we say that (1.5) is null-controllable in time $T$. In (1.5), $-\varepsilon y_{x x}$ represents a viscous term. Indeed, if $v_{\varepsilon}=0$, we have that

$$
\frac{\mathrm{d}}{\mathrm{d} t} \int_{0}^{\pi}|y(t, x)|^{2} \mathrm{~d} x=-2 \varepsilon \int_{0}^{\pi}\left|y_{x}(t, x)\right|^{2} \mathrm{~d} x \leq 0 \quad \forall t \geq 0,
$$

which means that the $L^{2}$-norm of the solution $y$ of (1.5) is decreasing in time.

Equation (1.5) is known as the linear complex Ginzburg-Landau equation. With a cubic nonlinearity, it plays an important role in the theory of amplitude equations and provides a simple model for turbulence (see, for instance, $[4,21])$. At the same time (1.5) may be viewed as a Schrödinger equation with an added viscous term. As such it models an array of optical fibers in a weakly lossy medium (see [29]). The boundary controllability properties for the cubic complex Ginzburg-Landau equation have been studied in [28], in a general domain, by means of an appropriate Carleman inequality. For the linear problem, interior controllability results have been obtained in $[9,10]$ whereas [1] is concerned with the stabilization around an unstable equilibrium state.

In this article we are interested in the following issue: Given $T>0, \varepsilon>0$ and $y^{0} \in H^{-1}(0, \pi)$, is there a control $v_{\varepsilon} \in L^{2}(0, T)$ for (1.5) such that the family $\left(v_{\varepsilon}\right)_{\varepsilon>0}$ converges to a control of (1.1) when $\varepsilon \rightarrow 0$ ? Our main result reads as follows.

Theorem 1.1. There exists $T>0$ with the property that, for any $y^{0} \in H^{-1}(0, \pi)$ and $\varepsilon \in(0,1]$, there exists a control $v_{\varepsilon} \in L^{2}(0, T)$ of $(1.5)$ such that the family $\left(v_{\varepsilon}\right)_{\varepsilon \in(0,1]}$ is uniformly bounded in $L^{2}(0, T)$ and any weak limit $v$ of it is a control in time $T$ for (1.1).

We emphasize that the focus of our concern is the uniform controllability with respect to $\varepsilon$ of (1.5) and the possibility of obtaining controls for (1.1) as limits, when $\varepsilon$ goes to zero, of controls for (1.5). The interest of this problem is justified by the use of the vanishing viscosity as a typical mechanism to study Cauchy problems and to improve convergence of numerical schemes for hyperbolic conservation laws and shocks. For instance, in $[13,14]$, it is proved that, by adding an extra numerical viscosity term, the dispersive properties of the finite 
difference scheme for the nonlinear Schrödinger equation become uniform when the mesh-size tends to zero. This scheme reproduces at the discrete level the properties of the continuous Schrödinger equation by dissipating the high frequency numerical spurious solutions. On the other hand, a viscosity term is introduced in [8] to prove the existence of solutions of hyperbolic equations. In both examples the viscosity is devoted to tend to zero in order to obtain the original system. Thus, a legitimate question is related to the behavior and the sensitivity of the controls during this process.

The method we employ in this article is different from the one used in [20,28]. It is based on the construction of an explicit control $v_{\varepsilon}$ for (1.5) such that the family $\left(v_{\varepsilon}\right)_{\varepsilon \in(0,1]}$ is uniformly bounded in $L^{2}(0, T)$. Nextly, a classical (weak) limit argument, allow us to obtain the desired result. The controls $\left(v_{\varepsilon}\right)_{\varepsilon \in(0,1]}$ are given in terms of a biorthogonal sequence $\left(\zeta_{m}\right)_{m \geq 1}$ to the family of exponential functions $\left(\mathrm{e}^{\mu_{n} t}\right)_{n \geq 1}$, where $\mu_{n}=\mu_{n}(\varepsilon)=\varepsilon n^{2}-\mathrm{i} n^{2}$ are the eigenvalues of the differential operator corresponding to the perturbed equation (1.5). More precisely, a control $v_{\varepsilon}$ for the initial datum $y^{0}(x)=\sum_{n \geq 1} a_{n} \sin (n x)$ of (1.5) is obtained from a formula similar to (1.4):

$$
v_{\varepsilon}(t)=\sum_{n=1}^{\infty} \frac{(-1)^{n} \pi a_{n}}{2 n(\mathrm{i}+\varepsilon)} \mathrm{e}^{-\frac{T}{2} \bar{\mu}_{n}} \zeta_{n}\left(t-\frac{T}{2}\right) \quad \forall t \in(0, T) .
$$

Our construction allows to prove that, if $T>0$ is sufficiently large but independent of $\varepsilon$, the following nonstandard Ingham type inequality holds

$$
\sum_{n \geq 1}\left|a_{n}\right|^{2} \mathrm{e}^{-2 \alpha\left|\Re\left(\mu_{n}\right)\right|} \leq C(T) \int_{-\frac{T}{2}}^{\frac{T}{2}}\left|\sum_{n \geq 1} a_{n} \mathrm{e}^{\mu_{n} t}\right|^{2} \mathrm{~d} t \quad \forall\left(a_{n}\right)_{n \geq 1} \in \ell^{2},
$$

where $\alpha$ and $C(T)$ are positive constants independent of $\varepsilon$. Note that, although (1.8) looks like (1.3), its proof cannot be obtained as in [16] because of the different nature of the exponents $\mu_{n}$ which are no longer purely imaginary numbers. The negative exponential weights from the left hand side of (1.8) reflect the particular form of our exponents.

Through the paper, if $\lambda$ is a complex number, $\Re(\lambda)$ and $\Im(\lambda)$ will denote its real and imaginary part, respectively. Moreover $[a]$ will represent the greatest integer smaller than the real number $a$.

Due to the character of equation (1.5), our controllability problem belongs to the interface between parabolic and hyperbolic equations. From this point of view, it is related to $[7,12]$, where the controllability of the transport equation is consider by introducing a vanishing viscosity term. In [7] Carleman estimates are used to obtain a uniform bound for the family of controls. The same result is shown in [12], improving the control time, by means of nonharmonic Fourier analysis and biorthogonal technique. The recent article [19] deals with a nonlinear scalar conservation law perturbed by a small viscosity term and proves the uniform boundedness of the boundary controls.

The main difficulty in this type of problems consists of obtaining a uniform bound for the series (1.7). In the high frequencies range, the exponentially large norms $\left\|\zeta_{m}\right\|_{L^{2}}$ are compensated by the strong damping represented by the small negative exponential factors $\mathrm{e}^{-\frac{T}{2} \bar{\mu}_{n}}$. On the other hand, to the weakly dissipated low frequencies correspond norms $\left\|\zeta_{m}\right\|_{L^{2}}$ which are much smaller. This mechanism ensures the convergence of series (1.7) and the uniformly boundedness of the controls $\left(v_{\varepsilon}\right)_{\varepsilon \in(0,1]}$ for a large class of coefficients $\left(a_{n}\right)_{n \geq 1}$. However, in order to fully justify it, precise estimates of the biorthogonal's norm $\left\|\zeta_{m}\right\|_{L^{2}}$ on both $\varepsilon$ and $m$ are needed. This represents one of the main tasks of our work.

In $[9,10]$ it is studied an interior controllability problem for an equation whose principal part is

$$
\mathcal{P}(z)=(\alpha+\mathrm{i} \beta) \partial_{t} z+\sum_{j, k=1}^{N} \partial_{k}\left(a^{j k} \partial_{j} z\right)
$$


where $\alpha$ and $\beta$ are real functions. A pointwise weighted identity allows to deduce Carleman estimates and controllability results for the corresponding equation. The author is mainly concerned with the case $\alpha<0$, when uniform estimates with respect to $\beta$ are obtained. This allows to prove a uniform controllability for the perturbed heat equation. From this point of view, [10] is related to [22,33], where the null-controllability of the heat equation is obtained as a singular limit of the exact controllability property of a damped wave equation. This is complementary to our study where the dissipation vanishes and the singular limit is a conservative system.

The rest of the paper is organized as follows. Section 2 gives the equivalent characterization of the controllability property in terms of a moment problem. In Section 3, a biorthogonal sequence is constructed and evaluated. Section 4 is devoted to the proof of the main result and some final comments are given in Section 5.

\section{The MOMENT PROBLEM}

For the sake of completeness, we first present the main result concerning the well-posedness of (1.5).

Theorem 2.1. Given any $T>0, \varepsilon \geq 0, v \in L^{2}(0, T)$ and $y^{0} \in H^{-1}(0, \pi)$, there exists a unique weak solution $y \in C\left([0, T], H^{-1}(0, \pi)\right)$ of the problem

$$
\left\{\begin{array}{l}
y_{t}(t, x)-\mathrm{i} y_{x x}(t, x)-\varepsilon y_{x x}(t, x)=0, \quad x \in(0, \pi), t \in(0, T) \\
y(t, 0)=0, y(t, \pi)=v(t), \quad t>0 \\
y(0, x)=y^{0}(x), \quad x \in(0, \pi)
\end{array}\right.
$$

Proof. It is the same as for the nonhomogeneous Schrödinger equation and we omit it (see, for instance, [30], pp. 343-344).

We can give now the characterization of the controllability property of (1.5) in terms of a moment problem. Based on Fourier expansion of the solution, the moment problems have been widely used in linear control theory. We refer to $[2,18,32]$ for a detailed discussion of the subject.

Theorem 2.2. Let $T>0, \varepsilon \geq 0$ and $y^{0} \in H^{-1}(0, \pi)$ given by

$$
y^{0}(x)=\sum_{n=1}^{\infty} a_{n} \sin (n x)
$$

There exists a control $v_{\varepsilon} \in L^{2}(0, T)$ such that the solution $y$ of $(1.5)$ verifies $y(T)=0$ if, and only if, $v_{\varepsilon} \in L^{2}(0, T)$ is a solution of the moment problem

$$
\int_{-\frac{T}{2}}^{\frac{T}{2}} v_{\varepsilon}\left(s+\frac{T}{2}\right) \mathrm{e}^{s \bar{\mu}_{n}} \mathrm{~d} s=\frac{(-1)^{n} \pi}{2 n(\mathrm{i}+\varepsilon)} \mathrm{e}^{-\frac{T}{2} \bar{\mu}_{n}} a_{n} \quad \forall n \geq 1,
$$

where $\mu_{n}=\varepsilon n^{2}-\mathrm{i} n^{2}$.

Proof. We consider $\varphi^{0} \in H_{0}^{1}(0, \pi)$ and the following adjoint equation of (1.5)

$$
\left\{\begin{array}{l}
-\varphi_{t}(t, x)+\mathrm{i} \varphi_{x x}(t, x)-\varepsilon \varphi_{x x}(t, x)=0, \quad x \in(0, \pi), t \in(0, T) \\
\varphi(t, 0)=\varphi(t, \pi)=0, \quad t \in(0, T) \\
\varphi(T, x)=\varphi^{0}(x), \quad x \in(0, \pi)
\end{array}\right.
$$


We multiply $(1.5)$ by $\bar{\varphi}$ and integrate by parts over $(0, T) \times(0, \pi)$. It follows that $v_{\varepsilon} \in L^{2}(0, T)$ is a control for (1.5) if, and only if, it verifies

$$
\int_{0}^{T} v_{\varepsilon}(t) \bar{\varphi}_{x}(t, \pi) \mathrm{d} t=\frac{1}{\mathrm{i}+\varepsilon}\left\langle y^{0}, \bar{\varphi}(0)\right\rangle_{H^{-1}, H_{0}^{1}}
$$

for any $\varphi^{0} \in H_{0}^{1}(0, \pi)$ and $\varphi$ solution of $(2.3)$. Since $\{\sin (n x): n \geq 1\}$ is a basis of $H_{0}^{1}(0, \pi)$, we have to check (2.4) only for solutions of $(2.3)$ of the form $\varphi(t, x)=\mathrm{e}^{(t-T) \mu_{n}} \sin (n x), n \in \mathbb{N}^{*}$. Thus, $v_{\varepsilon}$ is a control for (1.5) if and only if it verifies (2.2).

The notion of biorthogonal is very useful in the study of moment problems like (2.2). We recall that $\left(\zeta_{m}\right)_{m \geq 1} \subset L^{2}\left(-\frac{T}{2}, \frac{T}{2}\right)$ is a biorthogonal sequence to the family of exponential functions $\left(\mathrm{e}^{\mu_{n} t}\right)_{n \geq 1}$ in $L^{2}\left(-\frac{T}{2}, \frac{T}{2}\right)$ iff

$$
\int_{-\frac{T}{2}}^{\frac{T}{2}} \zeta_{m}(t) \mathrm{e}^{\bar{\mu}_{n} t} \mathrm{~d} t=\delta_{n m} \quad \forall n, m \geq 1 .
$$

It is easy to see from (2.2) that, if $\left(\zeta_{m}\right)_{m \geq 1}$ is a biorthogonal sequence to the family of exponential functions $\left(\mathrm{e}^{\mu_{n} t}\right)_{n \geq 1}$ in $L^{2}\left(-\frac{T}{2}, \frac{T}{2}\right)$, then a control $v_{\varepsilon}$ of $(1.5)$ is given by (1.7), provided that the right hand series converges in $L^{2}(0, T)$. Now, the main task is to show that there exists a biorthogonal sequence $\left(\zeta_{m}\right)_{m \geq 1}$ and to evaluate its norm in order to prove the convergence of the series from $(1.7)$ for each $y^{0} \in H^{-1}(0, \pi)$.

\section{Construction of a Biorthogonal sequence}

The aim of this section is to construct and evaluate an explicit biorthogonal sequence to the family $\left(\mathrm{e}^{\mu_{n} t}\right)_{n \geq 1}$ in $L^{2}\left(-\frac{T}{2}, \frac{T}{2}\right)$. In order to do that, we introduce a family $\Psi_{m}(z)$ of entire functions of exponential type such that $\Psi_{m}\left(\mathrm{i} \bar{\mu}_{n}\right)=\delta_{m n}$. Nextly, Paley-Wiener's Theorem gives us a biorthogonal family $\left(\theta_{m}\right)_{m \geq 1}$ as the inverse Fourier transforms of $\left(\Psi_{m}\right)_{m \geq 1}$. Each $\Psi_{m}$ is obtained from a Weierstrass product $P_{m}$ multiplied by a function $M_{m, \varepsilon}$ with a suitable behavior on the real axis. Such a method was used for the first time by Paley and Wiener [26]. The main difficulty in our analysis is to obtain good estimates for the behavior of $P_{m}$ on the real axis, to construct an appropriate multiplier $M_{m, \varepsilon}$ and to evaluate carefully $M_{m, \varepsilon}\left(\mathrm{i} \lambda_{m}\right)$ in order to ensure the boundedness of $\Psi_{m}$ on the real axis. Finally, from Plancherel's Theorem, we obtain the desired estimates for the norms $\left\|\theta_{m}\right\|_{L^{2}}$ and we can pass to study the absolute convergence of the series (1.7).

For the non-specialist reader let us recall that an entire function $f: \mathbb{C} \rightarrow \mathbb{C}$ is of exponential type $A>0$ if there exists a constant $B>0$ such that the following inequality holds

$$
|f(z)| \leq B \mathrm{e}^{A|z|} \quad \forall z \in \mathbb{C}
$$

If $g \in L^{1}(\mathbb{R})$, we denote by $\widehat{g}$ the Fourier transform of $g$ defined by

$$
\widehat{g}(z)=\frac{1}{\sqrt{2 \pi}} \int_{\mathbb{R}} g(t) \mathrm{e}^{-\mathrm{i} t z} \mathrm{~d} t
$$

and the following inversion relation holds for any $g, \widehat{g} \in L^{1}(\mathbb{R})$

$$
g(t)=\frac{1}{\sqrt{2 \pi}} \int_{\mathbb{R}} \widehat{g}(x) \mathrm{e}^{\mathrm{i} t x} \mathrm{~d} x .
$$

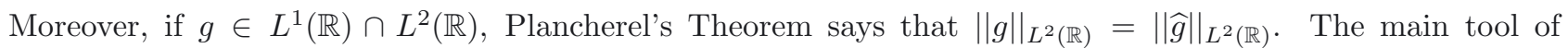
our analysis is Paley-Wiener's Theorem which we also recall for convenience. For its proof and other details concerning entire functions the interested reader is referred to [31]. 
Theorem (Paley-Wiener). Let $F$ be an entire function of exponential type $A$ with the property that $\int_{\mathbb{R}}|F(x)|^{2}$ $\mathrm{d} x<\infty$. Then there exists a function $f \in L^{2}(-A, A)$ such that

$$
\widehat{f}=F .
$$

Now, let us pass to construct our biorthogonal sequence. For any $m \in \mathbb{N}^{*}$, we define the function

$$
P_{m}(z)=\prod_{\substack{n \in \mathbb{Z}^{*} \\|n| \neq m}}\left(1-\frac{z}{\mathrm{i} \lambda_{n}}\right)\left(\frac{\lambda_{n}}{\lambda_{n}-\lambda_{m}}\right)
$$

where

$$
\lambda_{n}=\left\{\begin{array}{lll}
\text { in } n+\varepsilon n, & \text { if } n=q^{2} & \text { with } q \in \mathbb{N}^{*} \\
\mathrm{i} \sqrt{1+\varepsilon^{2}} n, & \text { if } n \neq q^{2}, n>0 & \text { with } q \in \mathbb{N}^{*} \\
\bar{\lambda}_{-n}, & \text { if } n<0 . &
\end{array}\right.
$$

Note that $\lambda_{q^{2}}=\bar{\mu}_{q}$, for any $q \geq 1$. Hence, the family $\left(\lambda_{m}\right)_{m \geq 1}$ is larger than $\left(\mu_{q}\right)_{q \geq 1}$. This extension of the family of exponents will be very important for the behavior on the real axis of $P_{m}$ (see the second comment from Sect. 5).

Lemma 3.1. For each $m \geq 1$ of the form $m=q^{2}$ with $q \in \mathbb{N}^{*}, P_{m}$ is an entire function of exponential type independent of $\varepsilon$ such that

$$
P_{m}\left(\mathrm{i} \lambda_{n}\right)=\delta_{m n}, \quad n \in \mathbb{N}^{*} .
$$

Proof. Properties (3.6) are evident if the product $P_{m}$ is convergent. In order to show the convergence and to study the behavior of $P_{m}$, we evaluate each of the following products

$$
R_{m}(z)=\prod_{\substack{n \in \mathbb{Z}^{*} \\|n| \neq m}}\left|1-\frac{z}{\mathrm{i} \lambda_{n}}\right| \text { and } Q_{m}=\prod_{\substack{n \in \mathbb{Z}^{*} \\|n| \neq m}}\left|\frac{\lambda_{n}}{\lambda_{n}-\lambda_{m}}\right| .
$$

For any $z \in \mathbb{C}$, we have that

$$
\begin{aligned}
R_{m}(z) & =\exp \left(\sum_{n=1}^{N_{z}} \ln \left|1-\frac{z^{2}}{\left|\lambda_{n}\right|^{2}}+2 \mathrm{i} z \Re\left(\frac{1}{\lambda_{n}}\right)\right|+\sum_{n=N_{z}}^{\infty} \ln \left|1-\frac{z^{2}}{\left|\lambda_{n}\right|^{2}}+2 \mathrm{i} z \Re\left(\frac{1}{\lambda_{n}}\right)\right|\right) \\
& =\exp (A(z)+B(z)),
\end{aligned}
$$

where $N_{z}$ is defined by

Then

$$
N_{z}=\max \left\{N \geq 1:\left|2 z \Re\left(\frac{1}{\lambda_{n}}\right)\right| \leq \frac{|z|^{2}}{\left|\lambda_{n}\right|^{2}}, \forall n \leq N\right\}
$$

Moreover, since

$$
A(z) \leq \sum_{n=1}^{N_{z}} \ln \left(1+\frac{2|z|^{2}}{\left|\lambda_{n}\right|^{2}}\right) \leq \sum_{n=1}^{N_{z}} \ln \left(1+\frac{2|z|^{2}}{n^{2}}\right) \leq \int_{0}^{\infty} \ln \left(1+\frac{2|z|^{2}}{s^{2}}\right) \mathrm{d} s=\sqrt{2} \pi|z| .
$$

we have that

$$
\sum_{n \geq 1}\left|\Re\left(\frac{1}{\lambda_{n}}\right)\right|=\sum_{n \geq 1} \frac{\varepsilon n^{2}}{\varepsilon^{2} n^{4}+n^{4}}=\frac{\varepsilon}{\varepsilon^{2}+1} \sum_{n \geq 1} \frac{1}{n^{2}} \leq \frac{2 \varepsilon}{1+\varepsilon^{2}},
$$

$$
B(z) \leq \sum_{n=N_{z}+1}^{\infty} \ln \left(1+4|z|\left|\Re\left(\frac{1}{\lambda_{n}}\right)\right|\right) \leq \sum_{n=N_{z}+1}^{\infty} 4|z|\left|\Re\left(\frac{1}{\lambda_{n}}\right)\right| \leq \frac{8 \varepsilon}{1+\varepsilon^{2}}|z| .
$$


Thus,

$$
R_{m}(z)=\prod_{\substack{n \in \mathbb{Z}^{*} \\|n| \neq m}}\left|1-\frac{z}{\mathrm{i} \lambda_{n}}\right| \leq \exp \left(\left(\sqrt{2} \pi+\frac{8 \varepsilon}{1+\varepsilon^{2}}\right)|z|\right) \quad \forall z \in \mathbb{C}
$$

Now, we pass to study $Q_{m}$. We have that

$$
\begin{aligned}
\left|Q_{m}\right|^{2} & =\prod_{\substack{n \in \mathbb{Z}^{*} \\
|n| \neq m}}\left|\frac{\lambda_{n}}{\lambda_{n}-\lambda_{m}}\right|^{2}=\prod_{\substack{n=1 \\
n \neq m}}^{\infty} \frac{\left|\lambda_{n}\right|^{4}}{\left.|| \lambda_{n}\right|^{2}+\lambda_{m}^{2}-\left.2 \lambda_{m} \Re\left(\lambda_{n}\right)\right|^{2}} \\
& =\frac{\left.|| \lambda_{m}\right|^{2}+\left.\lambda_{m}^{2}\right|^{2}}{\left|\lambda_{m}\right|^{4}} \prod_{n=1}^{\infty} \frac{\left|\lambda_{n}\right|^{4}}{\left.|| \lambda_{n}\right|^{2}+\left.\lambda_{m}^{2}\right|^{2}} \prod_{\substack{n=1 \\
n=k^{2}, n \neq m}}^{\infty} \frac{\left.|| \lambda_{n}\right|^{2}+\left.\lambda_{m}^{2}\right|^{2}}{\left.|| \lambda_{n}\right|^{2}+\lambda_{m}^{2}-\left.2 \lambda_{m} \Re\left(\lambda_{n}\right)\right|^{2}} \\
& =\frac{4 \varepsilon^{2}}{1+\varepsilon^{2}}\left|\frac{\frac{i \lambda_{m} \pi}{\sqrt{1+\varepsilon^{2}}}}{\sin \left(\frac{\mathrm{i} \lambda_{m} \pi}{\sqrt{1+\varepsilon^{2}}}\right)}\right|^{2} \prod_{\substack{n=1 \\
n=k^{2}, n \neq m}}^{\infty} \frac{\left.|| \lambda_{n}\right|^{2}+\left.\lambda_{m}^{2}\right|^{2}}{\left.|| \lambda_{n}\right|^{2}+\lambda_{m}^{2}-\left.2 \lambda_{m} \Re\left(\lambda_{n}\right)\right|^{2}}=Q_{m}^{1} Q_{m}^{2} .
\end{aligned}
$$

On the one hand we have that

$$
\begin{aligned}
Q_{m}^{1} & =\frac{4 \varepsilon^{2}}{1+\varepsilon^{2}}\left|\frac{\frac{i \lambda_{m} \pi}{\sqrt{1+\varepsilon^{2}}}}{\sin \left(\frac{\mathrm{i} \lambda_{m} \pi}{\sqrt{1+\varepsilon^{2}}}\right)}\right|^{2}=\frac{4 \pi^{2} \varepsilon^{2} m^{2}}{\left(1+\varepsilon^{2}\right)\left|\sin \left(-\frac{m \pi}{\sqrt{1+\varepsilon^{2}}}+\mathrm{i} \frac{m \pi}{\sqrt{1+\varepsilon^{2}}}\right)\right|^{2}} \\
& =\frac{16 \pi^{2} \varepsilon^{2} m^{2}}{\left(1+\varepsilon^{2}\right)\left(\mathrm{e}^{\frac{2 m \pi \varepsilon}{\sqrt{1+\varepsilon^{2}}}}+\mathrm{e}^{-\frac{2 m \pi \varepsilon}{\sqrt{1+\varepsilon^{2}}}}-2 \cos \left(\frac{2 m \pi \varepsilon}{\sqrt{1+\varepsilon^{2}}}\right)\right)} \leq \frac{16 \pi^{2} \varepsilon^{2} m^{2}}{\left(1+\varepsilon^{2}\right)\left(\mathrm{e}^{\frac{2 m \pi \varepsilon}{\sqrt{1+\varepsilon^{2}}}}+\mathrm{e}^{-\frac{2 m \pi \varepsilon}{\sqrt{1+\varepsilon^{2}}}}-2\right)} \\
& \leq \frac{16 \pi^{2} \varepsilon^{2} m^{2}}{1+\varepsilon^{2}} \times \frac{1+\varepsilon^{2}}{4 \pi^{2} \varepsilon^{2} m^{2}}=4 .
\end{aligned}
$$

On the other hand, since $m=q^{2}, q \in \mathbb{N}^{*}$, we have that

$$
\begin{aligned}
Q_{m}^{2} & =\prod_{\substack{n=1 \\
n=k^{2}, n \neq m}}^{\infty} \frac{\left.|| \lambda_{n}\right|^{2}+\left.\lambda_{m}^{2}\right|^{2}}{\left.|| \lambda_{n}\right|^{2}+\lambda_{m}^{2}-\left.2 \lambda_{m} \Re\left(\lambda_{n}\right)\right|^{2}} \\
& =\prod_{\substack{n=1 \\
n=k^{2}, n \neq m}}^{\infty} \frac{\left|\Re\left(\lambda_{n}\right)^{2}+\Im\left(\lambda_{n}\right)^{2}+\Re\left(\lambda_{m}\right)^{2}-\Im\left(\lambda_{m}\right)^{2}+2 i \Re\left(\lambda_{m}\right) \Im\left(\lambda_{m}\right)\right|^{2}}{\left.\mid\left(\lambda_{m}\right)-\Re\left(\lambda_{n}\right)\right)^{2}+\Im\left(\lambda_{n}\right)^{2}-\Im\left(\lambda_{m}\right)^{2}+\left.2 i \Im\left(\lambda_{m}\right)\left(\Re\left(\lambda_{m}\right)-\Re\left(\lambda_{n}\right)\right)\right|^{2}} \\
& =\prod_{\substack{n=1 \\
n=k^{2}, n \neq m}}^{\infty}\left(1+\frac{4 \varepsilon^{2} m n\left(n^{2}-n m+m^{2}\right)}{(m-n)^{2}\left((m+n)^{2}+\varepsilon^{2}(m-n)^{2}\right)}\right) \leq \prod_{\substack{n=1 \\
n=k^{2}, n \neq m}}^{\infty}\left(1+\frac{4 \varepsilon^{2} m n}{(m-n)^{2}}\right) \\
& \leq \exp \left(\sum_{k=1, k \neq q}^{\infty} \frac{4 \varepsilon^{2} q^{2} k^{2}}{\left(q^{2}-k^{2}\right)^{2}}\right) \leq \exp \left(\sum_{k=1, k \neq q}^{\infty} \frac{4 \varepsilon^{2} q^{2}}{(q-k)^{2}}\right) \leq \exp \left(\sum_{k=1}^{\infty} \frac{8 \varepsilon^{2} q^{2}}{k^{2}}\right) \leq \exp \left(16 \varepsilon^{2} q^{2}\right) .
\end{aligned}
$$

Hence,

$$
Q_{m}=\prod_{\substack{n \in \mathbb{Z}^{*} \\|n| \neq m}}\left|\frac{\lambda_{n}}{\lambda_{n}-\lambda_{m}}\right| \leq 4 \exp \left(16 \varepsilon \Re\left(\lambda_{m}\right)\right)
$$

This completes the proof of the lemma.

Now, we pass to study the behavior on the real axis of the function $P_{m}$. 
Lemma 3.2. For each $m \geq 1$ of the form $m=q^{2}$ with $q \in \mathbb{N}^{*}$, the function $P_{m}$ defined by (3.5) has the following property

$$
\left|P_{m}(x)\right| \leq C \exp \left(32 \varepsilon \sqrt{|x|}+16 \varepsilon \Re\left(\lambda_{m}\right)\right) \quad \forall x \in \mathbb{R},
$$

where $C$ is a positive constant, independent of $\varepsilon$ and $m$.

Proof. In order to establish the behavior of $P_{m}$ on the real axis we consider

$$
\begin{aligned}
\left|R_{m}(x)\right|^{2} & =\prod_{\substack{n \in \mathbb{Z}^{*} \\
|n| \neq m}}\left|1-\frac{x}{\mathrm{i} \lambda_{n}}\right|^{2}=\prod_{\substack{n \in \mathbb{N}^{*} \\
n \neq m}} \frac{\left(|x|^{2}-\left|\lambda_{n}\right|^{2}\right)^{2}+4 x^{2} \Re^{2}\left(\lambda_{n}\right)}{\left|\lambda_{n}\right|^{4}} \\
& =\prod_{\substack{n \in \mathbb{N}^{*} \\
n \neq m}} \frac{\left(|x|^{2}-\left|\lambda_{n}\right|^{2}\right)^{2}}{\left|\lambda_{n}\right|^{4}} \prod_{\substack{n \in \mathbb{N}^{*} \\
n \neq m}}\left(1+\frac{4 x^{2} \Re^{2}\left(\lambda_{n}\right)}{\left(|x|^{2}-\left|\lambda_{n}\right|^{2}\right)^{2}}\right)=R_{m}^{1}(x) R_{m}^{2}(x) .
\end{aligned}
$$

First, we evaluate

$$
\begin{aligned}
R_{m}^{1}(x) & =\prod_{\substack{n \in \mathbb{N}^{*} \\
n \neq m}} \frac{\left(|x|^{2}-\left|\lambda_{n}\right|^{2}\right)^{2}}{\left|\lambda_{n}\right|^{4}}=\prod_{\substack{n \in \mathbb{N}^{*} \\
n \neq m}} \frac{\left(|x|^{2}-\left(1+\varepsilon^{2}\right) n^{2}\right)^{2}}{\left(1+\varepsilon^{2}\right)^{2} n^{4}} \\
& =\frac{\left(1+\varepsilon^{2}\right)^{2} m^{4}}{\left(|x|^{2}-\left(1+\varepsilon^{2}\right) m^{2}\right)^{2}} \prod_{n \in \mathbb{N}^{*}} \frac{\left(|x|^{2}-\left(1+\varepsilon^{2}\right) n^{2}\right)^{2}}{\left(1+\varepsilon^{2}\right)^{2} n^{4}}=\left(\frac{m^{2}}{m^{2}-\frac{x^{2}}{1+\varepsilon^{2}}}\right)^{2}\left(\frac{\sin \left(\frac{\pi|x|}{\sqrt{1+\varepsilon^{2}}}\right)}{\frac{\pi|x|}{\sqrt{1+\varepsilon^{2}}}}\right)^{2} .
\end{aligned}
$$

Now, we study

$$
\begin{aligned}
R_{m}^{2}(x) & =\prod_{\substack{n \in \mathbb{N}^{*} \\
n \neq m}}\left(1+\frac{4 x^{2} \Re^{2}\left(\lambda_{n}\right)}{\left(|x|^{2}-\left|\lambda_{n}\right|^{2}\right)^{2}}\right)=\frac{\left(|x|^{2}-\left|\lambda_{m}\right|^{2}\right)^{2}}{\left(|x|^{2}-\left|\lambda_{m}\right|^{2}\right)^{2}+4 x^{2} \Re^{2}\left(\lambda_{m}\right)} \prod_{n \in \mathbb{N}^{*}}\left(1+\frac{4 x^{2} \Re^{2}\left(\lambda_{n}\right)}{\left(|x|^{2}-\left|\lambda_{n}\right|^{2}\right)^{2}}\right) \\
& =\frac{\left(|x|^{2}-\left|\lambda_{m}\right|^{2}\right)^{2}}{\left(|x|^{2}-\left|\lambda_{m}\right|^{2}\right)^{2}+4 x^{2} \Re^{2}\left(\lambda_{m}\right)} \prod_{k \in \mathbb{N}^{*}}\left(1+\frac{4 \varepsilon^{2}}{1+\varepsilon^{2}} \frac{t^{4} k^{4}}{\left(t^{4}-k^{4}\right)^{2}}\right),
\end{aligned}
$$

where we have used the notation $t=\frac{\sqrt{x}}{\sqrt[4]{1+\varepsilon^{2}}} \cdot$ It follows that

$$
\begin{aligned}
R_{m}^{2}(x) \leq & \frac{\left(|x|^{2}-\left|\lambda_{m}\right|^{2}\right)^{2}}{\left(|x|^{2}-\left|\lambda_{m}\right|^{2}\right)^{2}+4 x^{2} \Re^{2}\left(\lambda_{m}\right)} \prod_{k \in \mathbb{N}^{*}}\left(1+\frac{4 \varepsilon^{2} t^{2}}{(t-k)^{2}}\right) \\
= & \frac{\left(|x|^{2}-\left|\lambda_{m}\right|^{2}\right)^{2}}{\left(|x|^{2}-\left|\lambda_{m}\right|^{2}\right)^{2}+4 x^{2} \Re^{2}\left(\lambda_{m}\right)} \exp \left(\sum_{k=1}^{[t]} \ln \left(1+\frac{4 \varepsilon^{2} t^{2}}{(t-k)^{2}}\right)+\sum_{k=[t]+1}^{\infty} \ln \left(1+\frac{4 \varepsilon^{2} t^{2}}{(k-t)^{2}}\right)\right) \\
\leq & \frac{\left(|x|^{2}-\left|\lambda_{m}\right|^{2}\right)^{2}}{\left(|x|^{2}-\left|\lambda_{m}\right|^{2}\right)^{2}+4 x^{2} \Re^{2}\left(\lambda_{m}\right)}\left(1+\frac{4 \varepsilon^{2} t^{2}}{(t-[t])^{2}}\right)\left(1+\frac{4 \varepsilon^{2} t^{2}}{([t]+1-t)^{2}}\right) \\
& \times \exp \left(\int_{0}^{t} \ln \left(1+\frac{4 \varepsilon^{2} t^{2}}{(t-s)^{2}}\right) \mathrm{d} s+\int_{t}^{\infty} \ln \left(1+\frac{4 \varepsilon^{2} t^{2}}{(s-t)^{2}}\right) \mathrm{d} s\right) .
\end{aligned}
$$


We evaluate now each of the integrals appearing at the exponent. We have that

$$
\begin{aligned}
I_{1} & =\int_{0}^{t} \ln \left(1+\frac{4 \varepsilon^{2} t^{2}}{(t-s)^{2}}\right) \mathrm{d} s=-\int_{0}^{t}(t-s)^{\prime} \ln \left(1+\frac{4 \varepsilon^{2} t^{2}}{(t-s)^{2}}\right) \mathrm{d} s \\
& =t \ln \left(1+4 \varepsilon^{2}\right)+\int_{0}^{t} \frac{8 \varepsilon^{2} t^{2}}{(t-s)^{2}+4 \varepsilon^{2} t^{2}} \mathrm{~d} s \\
I_{2} & =\int_{t}^{\infty} \ln \left(1+\frac{4 \varepsilon^{2} t^{2}}{(s-t)^{2}}\right) \mathrm{d} s=\int_{t}^{\infty}(s-t)^{\prime} \ln \left(1+\frac{4 \varepsilon^{2} t^{2}}{(s-t)^{2}}\right) \mathrm{d} s \\
& =\int_{t}^{\infty} \frac{8 \varepsilon^{2} t^{2}}{(s-t)^{2}+4 \varepsilon^{2} t^{2}} \mathrm{~d} s
\end{aligned}
$$

and consequently

$$
\begin{aligned}
I_{1}+I_{2} & =t \ln \left(1+4 \varepsilon^{2}\right)+\int_{0}^{\infty} \frac{8 \varepsilon^{2} t^{2}}{(t-s)^{2}+4 \varepsilon^{2} t^{2}} \mathrm{~d} s \\
& \leq t \ln \left(1+4 \varepsilon^{2}\right)+8 \varepsilon^{2} t^{2}\left(\int_{0}^{(1-2 \varepsilon) t} \frac{\mathrm{d} s}{(t-s)^{2}}+\int_{(1+2 \varepsilon) t}^{\infty} \frac{\mathrm{d} s}{(t-s)^{2}}+\int_{(1-2 \varepsilon) t}^{(1+2 \varepsilon) t} \frac{\mathrm{d} s}{4 \varepsilon^{2} t^{2}}\right) \\
& =t \ln \left(1+4 \varepsilon^{2}\right)+8 \varepsilon^{2} t^{2}\left(\frac{1}{2 \varepsilon t}-\frac{1}{t}+\frac{1}{2 \varepsilon t}+\frac{1}{\varepsilon t}\right) \leq 16 \varepsilon t
\end{aligned}
$$

We deduce that

$$
\begin{aligned}
R_{m}^{2}(x) \leq & \frac{\left(|x|^{2}-\left|\lambda_{m}\right|^{2}\right)^{2}}{\left(|x|^{2}-\left|\lambda_{m}\right|^{2}\right)^{2}+4 x^{2} \Re^{2}\left(\lambda_{m}\right)}\left(1+\frac{4 \varepsilon^{2} x}{\left(\sqrt{x}-\sqrt[4]{1+\varepsilon^{2}}\left[\frac{\sqrt{x}}{\sqrt[4]{1+\varepsilon^{2}}}\right]\right)^{2}}\right) \\
& \times\left(1+\frac{4 \varepsilon^{2} x}{\left(\sqrt[4]{1+\varepsilon^{2}}\left(\left[\frac{\sqrt{x}}{\sqrt[4]{1+\varepsilon^{2}}}\right]+1\right)-\sqrt{x}\right)^{2}}\right) \exp \left(\frac{16 \varepsilon \sqrt{x}}{\sqrt[4]{1+\varepsilon^{2}}}\right)
\end{aligned}
$$

Finally, we have

$$
\begin{aligned}
\left|R_{m}(x)\right|^{2} & \leq \frac{\left(\left(1+\varepsilon^{2}\right) m^{2}\right)^{2}}{\left(|x|^{2}-\left(1+\varepsilon^{2}\right) m^{2}\right)^{2}+4 x^{2} \varepsilon^{2} m^{2}}\left(\frac{\sin \pi \frac{|x|}{\sqrt{1+\varepsilon^{2}}}}{\pi \frac{|x|}{\sqrt{1+\varepsilon^{2}}}}\right)^{2}\left(1+\frac{4 \varepsilon^{2} x}{\left(\sqrt{x}-\sqrt[4]{1+\varepsilon^{2}}\left[\frac{\sqrt{x}}{\sqrt[4]{1+\varepsilon^{2}}}\right]\right)^{2}}\right) \\
& \times\left(1+\frac{4 \varepsilon^{2} x}{\left(\sqrt[4]{1+\varepsilon^{2}}\left(\left[\frac{\sqrt{x}}{\sqrt[4]{1+\varepsilon^{2}}}\right]+1\right)-\sqrt{x}\right)^{2}}\right) \exp \left(\frac{16 \varepsilon \sqrt{x}}{\sqrt[4]{1+\varepsilon^{2}}}\right) \leq C \exp (16 \varepsilon \sqrt{|x|}) .
\end{aligned}
$$

Note that the numerators $\sqrt{x}-\sqrt[4]{1+\varepsilon^{2}}\left[\frac{\sqrt{x}}{\sqrt[4]{1+\varepsilon^{2}}}\right]$ or $\sqrt{x}-\sqrt[4]{1+\varepsilon^{2}}\left(1+\left[\frac{\sqrt{x}}{\sqrt[4]{1+\varepsilon^{2}}}\right]\right)$ vanishes if and only if $\frac{|x|}{\sqrt{1+\varepsilon^{2}}}=l^{2}, l \in \mathbb{N}$. Taking into account that

$$
\left|\sin \left(\pi \frac{|x|}{\sqrt{1+\varepsilon^{2}}}\right)\right|=\left|\sin \pi\left(\frac{|x|}{\sqrt{1+\varepsilon^{2}}}-l^{2}\right)\right|=\left|\sin \pi\left(\frac{\sqrt{|x|}}{\sqrt[4]{1+\varepsilon^{2}}}-l\right)\left(\frac{\sqrt{|x|}}{\sqrt[4]{1+\varepsilon^{2}}}+l\right)\right|
$$

and using the fact that $|\sin (x)| \leq|x|$ it is easy to show the existence of the bound $C$ in $(3.10)$. 
On the other hand, the expression $\left(|x|^{2}-\left(1+\varepsilon^{2}\right) m^{2}\right)^{2}+4 x^{2} \varepsilon^{2} m^{2}$ attains its minimum when $\frac{|x|}{\sqrt{1+\varepsilon^{2}}}=m$. In this case the first term from the right hand side of (3.10) is $\frac{1}{\varepsilon^{2}}$ and will be compensated by $4 \varepsilon^{2} x$ from the third or forth right hand side term.

From (3.8) and (3.10), we deduce that inequality (3.9) holds and the proof is finished.

In order to complete the construction of the biorthogonal sequence we have to find a multiplier to compensate the growth (3.9) of $P_{m}$ on the real axis. We use an idea of Ingham [15], generalized by Redheffer [27].

Lemma 3.3. For any $\varepsilon \in(0,1]$ and $m \geq 1$ of the form $m=q^{2}$ with $q \in \mathbb{N}^{*}$, there exists a function $M_{m, \varepsilon}: \mathbb{C} \rightarrow \mathbb{C}$ such that:

(1) $M_{m, \varepsilon}$ is an entire function of exponential type independent of $\varepsilon$ and $m$;

(2) $M_{m, \varepsilon}(x) \leq \exp (-\varepsilon \sqrt{|x|})$ for all $x \in \mathbb{R}$;

(3) $M_{m, \varepsilon}\left(\mathrm{i} \lambda_{m}\right) \geq C \exp \left(-R\left|\Re\left(\lambda_{m}\right)\right|\right)$,

where $C$ and $R$ are positive constants independent of $\varepsilon$ and $m$.

Proof. Let $\left(\rho_{n}\right)_{n \geq 1}$ be the nonincreasing sequence defined by

$$
\rho_{n}=\frac{\varepsilon e}{n^{3 / 2}} \quad \forall n \geq 1
$$

Note that $\sum_{n \geq 1} \rho_{n}:=\varepsilon \ell<\infty$. The function $M_{m, \varepsilon}$ defined by

$$
M_{m, \varepsilon}(z)=\mathrm{e}^{-2-\delta \varepsilon m} \prod_{n \geq n_{0}} \frac{\sin \left(\rho_{n} z\right)}{\rho_{n} z}
$$

is an entire function of exponential type less than $\varepsilon \ell$. The numbers $n_{0} \in \mathbb{N}^{*}$ and $\delta>0$ will be conveniently chosen latter on.

We pass to evaluate $M_{m, \varepsilon}(x)$. We shall consider three cases:

- $0 \leq \varepsilon \sqrt{|x|}<1$. In this case

$$
\left|M_{m, \varepsilon}(x)\right|=\mathrm{e}^{-2-\delta \varepsilon m} \prod_{n \geq n_{0}} \frac{\sin \left(\rho_{n}|x|\right)}{\rho_{n}|x|} \leq \mathrm{e}^{-1} \leq \mathrm{e}^{-\varepsilon \sqrt{|x|}} .
$$

- $1 \leq \varepsilon \sqrt{|x|} \leq n_{0}$. In this case

$$
\left|M_{m, \varepsilon}(x)\right|=\mathrm{e}^{-2-\delta \varepsilon m} \prod_{n \geq n_{0}} \frac{\sin \left(\rho_{n}|x|\right)}{\rho_{n}|x|} \leq \mathrm{e}^{-1-\delta \varepsilon m} \leq \mathrm{e}^{n_{0}-1-\delta \varepsilon m} \mathrm{e}^{-\varepsilon \sqrt{|x|}} .
$$

- $\varepsilon \sqrt{|x|}>n_{0}$. For any $p \geq n_{0}$, we have that

$$
\left|M_{m, \varepsilon}(x)\right|=\mathrm{e}^{-2-\delta \varepsilon m} \prod_{n \geq n_{0}} \frac{\sin \left(\rho_{n}|x|\right)}{\rho_{n}|x|} \leq \mathrm{e}^{-2-\delta \varepsilon m}\left(\frac{1}{\rho_{p}|x|}\right)^{p-n_{0}} \leq \mathrm{e}^{-p-2+n_{0}-\delta \varepsilon m}\left(\frac{p^{3 / 2}}{\varepsilon|x|}\right)^{p-n_{0}} .
$$

If we choose $p=[\varepsilon \sqrt{|x|}]>n_{0}$, since $|x|>\frac{n_{0}^{2}}{\varepsilon^{2}} \geq n_{0}^{2} \geq 1$, it follows that

$$
\frac{p^{3 / 2}}{\varepsilon|x|} \leq \frac{(\varepsilon \sqrt{|x|})^{3 / 2}}{\varepsilon|x|}=\sqrt[4]{\frac{\varepsilon^{2}}{|x|}} \leq \sqrt{\varepsilon} \leq 1
$$


Thus, since $p+1>\varepsilon \sqrt{|x|}$, we obtain that

$$
\left|M_{m, \varepsilon}(x)\right| \leq \mathrm{e}^{n_{0}-1-\delta \varepsilon m} \mathrm{e}^{-\varepsilon \sqrt{|x|}}
$$

From the previous estimates it follows that

$$
\left|M_{m, \varepsilon}(x)\right| \leq \max \left\{1, \mathrm{e}^{n_{0}-1-\delta \varepsilon m}\right\} \exp (-\varepsilon \sqrt{|x|}) \quad \forall x \in \mathbb{R} .
$$

If we denote $n_{1}=\inf \left\{n \geq 0:\left|\lambda_{m} \rho_{n}\right|<1\right\}$ it follows that $n_{1}=\left[\sqrt[3]{\varepsilon^{2}\left(1+\varepsilon^{2}\right) \mathrm{e}^{2} m^{2}}\right]$. In what follows we chose

$$
\begin{aligned}
& n_{0}=[\delta \varepsilon m]+1, \\
& \delta=\sqrt[3]{\mathrm{e}^{2}\left(1+\varepsilon^{2}\right)} .
\end{aligned}
$$

Note that, with this choice, $n_{0} \leq \delta \varepsilon m+1$. From (3.13), it follows that $M_{m, \varepsilon}(x)$ verifies the second property. Moreover,

$$
n_{1} \leq \max \left\{\delta(\varepsilon m)^{2 / 3}-1,1\right\} \leq \max \{\delta \varepsilon m, 1\} \leq n_{0} .
$$

Now, we pass to evaluate $M_{m, \varepsilon}\left(\mathrm{i} \lambda_{m}\right)$. Firstly, we remark that, for any $z \in \mathbb{C}$ with $|z|<1$,

$$
\ln \left|\frac{\sin z}{z}\right| \geq \ln \left(\frac{\sin |z|}{|z|}\right) \geq \ln \left(1-\frac{1}{6}|z|^{2}\right) \geq-\frac{1}{3}|z|^{2}
$$

From (3.16) it follows that $\left|\mathrm{i} \rho_{n} \lambda_{m}\right|<1$ for any $n \geq n_{0}$ and inequality (3.17) allows to evaluate the product from $\left|M_{m, \varepsilon}\left(\mathrm{i} \lambda_{m}\right)\right|$ as follows

$$
\begin{aligned}
& \prod_{n=n_{0}}^{\infty}\left|\frac{\sin \left(\mathrm{i} \rho_{n} \lambda_{m}\right)}{\mathrm{i} \rho_{n} \lambda_{m}}\right|=\exp \left(\sum_{n=n_{0}}^{\infty} \ln \left|\frac{\sin \left(\mathrm{i} \rho_{n} \lambda_{m}\right)}{\mathrm{i} \rho_{n} \lambda_{m}}\right|\right) \\
& \geq \exp \left(-\sum_{n=n_{0}}^{\infty} \frac{\left|\rho_{n}\right|^{2}\left|\lambda_{m}\right|^{2}}{3}\right) \geq \exp \left(-\frac{\mathrm{e}^{2} \varepsilon^{2}\left|\lambda_{m}\right|^{2}}{3}\left(\frac{1}{n_{0}^{3}}+\int_{[\delta \varepsilon m]+1}^{\infty} \frac{\mathrm{d} s}{s^{3}}\right)\right) \\
& =\exp \left(-\frac{\mathrm{e}^{2} \varepsilon^{2}\left|\lambda_{m}\right|^{2}}{3}\left(\frac{1}{([\delta \varepsilon m]+1)^{3}}+\frac{1}{2([\delta \varepsilon m]+1)^{2}}\right)\right) \geq \exp \left(-\frac{\mathrm{e}^{2} \varepsilon^{2}\left|\lambda_{m}\right|^{2}}{3} \frac{[\delta \varepsilon m]+3}{2([\delta \varepsilon m]+1)^{3}}\right) .
\end{aligned}
$$

Now, remark that

$$
\frac{\mathrm{e}^{2} \varepsilon^{2}\left|\lambda_{m}\right|^{2}}{3} \frac{[\delta \varepsilon m]+3}{2([\delta \varepsilon m]+1)^{3}} \leq \frac{\mathrm{e}^{2} \varepsilon^{2}\left|\lambda_{m}\right|^{2}}{6(\delta \varepsilon m)^{2}} \frac{[\delta \varepsilon m]+3}{[\delta \varepsilon m]+1}=\frac{\delta}{6} \frac{[\delta \varepsilon m]+3}{[\delta \varepsilon m]+1} \leq \frac{\delta}{2}
$$

and consequently

$$
\prod_{n=n_{0}}^{\infty}\left|\frac{\sin \left(\mathrm{i} \rho_{n} \lambda_{m}\right)}{\mathrm{i} \rho_{n} \lambda_{m}}\right| \geq \exp \left(-\frac{\delta}{2}\right) .
$$

From (3.18) we deduce immediately that

$$
\left|M_{m, \varepsilon}\left(\mathrm{i} \lambda_{m}\right)\right| \geq \exp \left(-\frac{\delta}{2}-2-\delta \varepsilon m\right) .
$$

From (3.19) it follows that the function $M_{m, \varepsilon}$ verifies the third property with $R=\delta_{0}$ and $C=\exp \left(-\frac{\delta_{0}}{2}-2\right)$, where $\delta_{0}=\sqrt[3]{2 \mathrm{e}^{2}}$, and the proof ends. 
Now, we have all the ingredients needed to show the existence of a biorthogonal sequence to the family $\left(\mathrm{e}^{\mu_{m} t}\right)_{m \in \mathbb{N}^{*}}$.

Theorem 3.1. There exists $T_{0}>0$ such that, for any $\varepsilon \in(0,1]$, we find a biorthogonal sequence $\left(\theta_{m}\right)_{m \in \mathbb{N}^{*}}$ to the family $\left(\mathrm{e}^{\mu_{m} t}\right)_{m \in \mathbb{N}^{*}}$ in $L^{2}\left(-\frac{T_{0}}{2}, \frac{T_{0}}{2}\right)$ with the following property

$$
\left\|\theta_{m}\right\|_{L^{2}\left(-\frac{T_{0}}{2}, \frac{T_{0}}{2}\right)} \leq C \exp \left(\alpha\left|\Re\left(\mu_{m}\right)\right|\right) \quad \forall m \geq 1
$$

where $C$ and $\alpha$ are positive constants independent of $\varepsilon$ and $m$.

Proof. For each $m \in \mathbb{N}^{*}$ we define the function

$$
\Psi_{m}(z)=P_{m^{2}}(z)\left(\frac{M_{m^{2}, \varepsilon}(z)}{M_{m^{2}, \varepsilon}\left(\mathrm{i} \lambda_{m^{2}}\right)}\right)^{32} \frac{\sin \left(\gamma\left(z-\mathrm{i} \lambda_{m^{2}}\right)\right)}{\gamma\left(z-\mathrm{i} \lambda_{m^{2}}\right)} \quad \forall z \in \mathbb{C},
$$

where $\gamma>0$ is an arbitrary positive constant independent of $\varepsilon$ and $m$.

From Lemmas 3.1 and 3.3 we deduce that there exists $T_{0}>0$ independent of $\varepsilon$ such that $\Psi_{m}$ is an entire function of exponential type $\frac{T_{0}}{2}$. In fact, we can choose $T_{0}$ any number greater than $2\left(\sqrt{2} \pi+\frac{8 \varepsilon}{1+\varepsilon^{2}}+32 \varepsilon \ell+\gamma\right)$. Moreover, from estimate (3.9) of the function $P_{m^{2}}$ on the real axis and the properties of the function $M_{m^{2}, \varepsilon}$ from Lemma 3.3, we obtain that

$$
\begin{aligned}
\left\|\Psi_{m}\right\|_{L^{2}(\mathbb{R})}^{2} & =\int_{-\infty}^{\infty}\left|\Psi_{m}(x)\right|^{2} \mathrm{~d} x \leq C^{2} \mathrm{e}^{32(R+\varepsilon)\left|\Re\left(\lambda_{m^{2}}\right)\right|} \int_{-\infty}^{\infty}\left|\frac{\sin \left(\gamma\left(x-\lambda_{m^{2}} \mathrm{i}\right)\right)}{\gamma\left(x-\lambda_{m^{2}} \mathrm{i}\right)}\right|^{2} \mathrm{~d} x \\
& =\frac{C^{2}}{\gamma} \mathrm{e}^{32(R+\varepsilon)\left|\Re\left(\lambda_{m^{2}}\right)\right|} \int_{-\infty}^{\infty}\left|\frac{\sin \left(t-\mathrm{i} \gamma \Re\left(\lambda_{m^{2}}\right)\right)}{t-\mathrm{i} \gamma \Re\left(\lambda_{m^{2}}\right)}\right|^{2} \mathrm{~d} t \leq C \mathrm{e}^{2 \alpha\left|\Re\left(\mu_{m}\right)\right|},
\end{aligned}
$$

where $\alpha$ is any number greater than $16 R+\gamma+16 \varepsilon$.

From Paley-Wiener's Theorem we deduce that there exists $\left(\theta_{m}\right)_{m \geq 1} \subset L^{2}\left(-\frac{T_{0}}{2}, \frac{T_{0}}{2}\right)$ such that

$$
\Psi_{m}(z)=\int_{-\frac{T_{0}}{2}}^{\frac{T_{0}}{2}} \theta_{m}(t) \mathrm{e}^{-\mathrm{i} z t} \mathrm{~d} x \quad \forall m \geq 1
$$

Since, for each $m \geq 1$, the functions $\Psi_{m}$ satisfy

$$
\Psi_{m}\left(\mathrm{i} \bar{\mu}_{n}\right)=\Psi_{m}\left(\mathrm{i} \lambda_{n^{2}}\right)=\delta_{m n} \quad \forall n \geq 1
$$

we have that $\left(\theta_{m}\right)_{m \geq 1}$ is a biorthogonal sequence to $\left(\mathrm{e}^{\mu_{m} t}\right)_{m \geq 1}$. Moreover, Plancherel's Theorem gives that (3.20) holds and the proof finishes.

The following theorem gives a new biorthogonal family to $\left(\mathrm{e}^{\mu_{m} t}\right)_{m \geq 1}$ with even better norm properties than those of the biorthogonal $\left(\theta_{m}\right)_{m \geq 1}$ from Theorem 3.1 (see, for instance, [17]).

Let $a>0$ and $k_{a}=\frac{\sqrt{2 \pi}}{a^{2}}\left(\chi_{a} * \chi_{a}\right)$, where $\chi_{a}$ represents the characteristic function $\chi_{[-a / 2, a / 2]}$. Evidently $\operatorname{supp}\left(k_{a}\right) \subset[-a, a]$. Also, we have

$$
\widehat{k}_{a}(\xi)=\frac{1}{\sqrt{2 \pi}} \int_{\mathbb{R}} k_{a}(t) \mathrm{e}^{-\mathrm{i} t \xi} \mathrm{d} t=\frac{2 \pi}{a^{2}} \widehat{\chi}_{a}(\xi) \widehat{\chi}_{a}(\xi)=\frac{4}{a^{2}} \frac{\sin ^{2}\left(\frac{\xi a}{2}\right)}{\xi^{2}}
$$

We define $\rho_{m}(x)=\mathrm{e}^{\mathrm{i} x \Im\left(\mu_{m}\right)} k_{a}(x), \operatorname{so} \operatorname{supp}\left(\rho_{m}\right) \subset[-a, a]$. 
Theorem 3.2. Let $\left(\theta_{m}\right)_{m \geq 1}$ be the biorthogonal in $L^{2}\left(-\frac{T_{0}}{2}, \frac{T_{0}}{2}\right)$ from Theorem 3.1 and $a>0$. We define

$$
\zeta_{m}=\frac{1}{2 \pi \widehat{\rho}_{m}\left(\mathrm{i} \bar{\mu}_{m}\right)} \theta_{m} * \rho_{m}, \quad \forall m \geq 1 .
$$

Then we have the following assertions:

(i) The family $\left(\zeta_{m}\right)_{m \geq 1}$ is a biorthogonal in $L^{2}\left(-\frac{T_{0}}{2}-a, \frac{T_{0}}{2}+a\right)$ to the family $\left(\mathrm{e}^{\mu_{m} t}\right)_{m \geq 1}$.

(ii) For any finite sequence $\left(\alpha_{m}\right)_{m \geq 1}$ we have

$$
\int_{-\frac{T_{0}}{2}-a}^{\frac{T_{0}}{2}+a}\left|\sum_{m \geq 1} \alpha_{m} \zeta_{m}(t)\right|^{2} \mathrm{~d} t \leq C\left(T_{0}, a\right) \sum_{m \geq 1}\left|\alpha_{m}\right|^{2} \exp \left(2 \alpha\left|\Re\left(\mu_{m}\right)\right|\right),
$$

where $\alpha$ is the same as in (3.20) and $C\left(T_{0}, a\right)$ is a constant depending only of $T_{0}$ and $a$.

Proof. (i) It is sufficient to remark that

$$
\int_{-\frac{T_{0}}{2}-a}^{\frac{T_{0}}{2}+a} \zeta_{m}(t) \mathrm{e}^{\bar{\mu}_{n} t} \mathrm{~d} t=\sqrt{2 \pi} \widehat{\zeta}_{m}\left(\mathrm{i} \bar{\mu}_{n}\right)=\frac{\widehat{\theta}_{m}\left(\mathrm{i} \bar{\mu}_{n}\right) \widehat{\rho}_{m}\left(\mathrm{i} \bar{\mu}_{n}\right)}{\widehat{\rho}_{m}\left(\mathrm{i} \bar{\mu}_{n}\right)}=\delta_{m n} .
$$

(ii) Firstly, we note that

$$
\widehat{\rho}_{m}\left(\mathrm{i} \bar{\mu}_{m}\right)=\frac{1}{\sqrt{2 \pi}} \int_{\mathbb{R}} \rho_{m}(t) \mathrm{e}^{t \bar{\mu}_{m}} \mathrm{~d} t=\frac{1}{\sqrt{2 \pi}} \int_{\mathbb{R}} \mathrm{e}^{-\mathrm{i} m^{2} t} k_{a}(t) \mathrm{e}^{t \bar{\mu}_{m}} \mathrm{~d} t=\widehat{k}_{a}\left(\mathrm{i} \varepsilon m^{2}\right) \geq 1 .
$$

Now, we have that

$$
\begin{aligned}
& \int_{-\frac{T_{0}}{2}-a}^{\frac{T_{0}}{2}+a}\left|\sum_{m \geq 1} \alpha_{m} \zeta_{m}(t)\right|^{2} \mathrm{~d} t=\int_{\mathbb{R}}\left|\sum_{m \geq 1} \alpha_{m} \widehat{\zeta}_{m}(t)\right|^{2} \mathrm{~d} t \\
& =\int_{\mathbb{R}}\left|\sum_{m \geq 1} \alpha_{m} \sqrt{2 \pi} \frac{\widehat{\theta}_{m}(t) \widehat{\rho}_{m}(t)}{\widehat{\rho}_{m}\left(\mathrm{i} \bar{\mu}_{n}\right)}\right|^{2} \mathrm{~d} t \leq 2 \pi \int_{\mathbb{R}}\left|\sum_{m \geq 1} \frac{\left|\alpha_{m}\right|}{\mid \widehat{\rho}_{m}\left(\mathrm{i} \bar{\mu}_{n}\right)}\left\|\widehat{\theta}_{m}\right\|_{L^{\infty}(\mathbb{R})}\right| \widehat{\rho}_{m}(t)||^{2} \mathrm{~d} t \\
& \leq \int_{\mathbb{R}}\left|\sum_{m \geq 1}\right| \alpha_{m}\left|\sqrt{T_{0}}\left\|\theta_{m}\right\|_{L^{2}\left(-\frac{T_{0}}{2}, \frac{T_{0}}{2}\right)}\right| \widehat{\rho}_{m}(t)||^{2} \mathrm{~d} t=\int_{\mathbb{R}}\left|\sum_{m \geq 1}\right| \alpha_{m}\left|\sqrt{T_{0}}\left\|\theta_{m}\right\|_{L^{2}\left(-\frac{T_{0}}{2}, \frac{T_{0}}{2}\right)}\right| \widehat{k}_{a}\left(t-m^{2}\right)||^{2} \mathrm{~d} t \\
& \leq \int_{-a}^{a} k_{a}^{2}(t)\left|\sum_{m \geq 1}\right| \alpha_{m}\left|\sqrt{T_{0}}\left\|\theta_{m}\right\|_{L^{2}\left(-\frac{T_{0}}{2}, \frac{T_{0}}{2}\right)} \mathrm{e}^{-\mathrm{i} m^{2} t}\right|^{2} \mathrm{~d} t \leq \frac{2 \pi}{a^{2}} T_{0} \int_{-a}^{a}\left|\sum_{m \geq 1}\right| \alpha_{m}\left|\left\|\theta_{m}\right\|_{L^{2}\left(-\frac{T_{0}}{2}, \frac{T_{0}}{2}\right)} \mathrm{e}^{-\mathrm{i} m^{2} t}\right|^{2} \mathrm{~d} t .
\end{aligned}
$$

By Ingham inequality (see [16]), there exists a positive constant $C$ depending only of $a$ such that

$$
\int_{-a}^{a}\left|\sum_{m \geq 1}\right| \alpha_{m}\left|\left\|\theta_{m}\right\|_{L^{2}\left(-\frac{T_{0}}{2}, \frac{T_{0}}{2}\right)} \mathrm{e}^{-\mathrm{i} m^{2} t}\right|^{2} \mathrm{~d} t \leq C \sum_{m \geq 1}\left|\alpha_{m}\right|^{2}\left\|\theta_{m}\right\|_{L^{2}\left(-\frac{T_{0}}{2}, \frac{T_{0}}{2}\right)} .
$$

Now, taking into account (3.20), we deduce immediately (3.24) and the proof ends. 
Remark 3.1. Let $T=T_{0}+2 a$ and $\left(\zeta_{m}\right)_{m \geq 1}$ be the biorthogonal in $L^{2}\left(-\frac{T}{2}, \frac{T}{2}\right)$ to the family $\left(\mathrm{e}^{\mu_{m} t}\right)_{m \geq 1}$ constructed in Theorem 3.2. By using the general biorthogonal properties and (3.24), we deduce that

$$
\begin{aligned}
& \sum_{n \geq 1}\left|a_{n}\right|^{2} \mathrm{e}^{-2 \alpha\left|\Re\left(\mu_{n}\right)\right|}=\int_{-\frac{T}{2}}^{\frac{T}{2}}\left(\sum_{n \geq 1} a_{n} \mathrm{e}^{-2 \alpha\left|\Re\left(\mu_{n}\right)\right|} \zeta_{n}(t)\right)\left(\sum_{n \geq 1} \bar{a}_{n} \mathrm{e}^{\bar{\mu}_{n} t}\right) \mathrm{d} t \\
& \leq\left(\int_{-\frac{T}{2}}^{\frac{T}{2}}\left|\sum_{n \geq 1} a_{n} \mathrm{e}^{-2 \alpha\left|\Re\left(\mu_{n}\right)\right|} \zeta_{n}(t)\right|^{2} \mathrm{~d} t\right)^{\frac{1}{2}}\left(\int_{-\frac{T}{2}}^{\frac{T}{2}}\left|\sum_{n \geq 1} \bar{a}_{n} \mathrm{e}^{\bar{\mu}_{n} t}\right|^{2} \mathrm{~d} t\right)^{\frac{1}{2}} \\
& \leq \sqrt{C\left(T_{0}, a\right)}\left(\sum_{n \geq 1}\left|a_{n}\right|^{2} \mathrm{e}^{-2 \alpha\left|\Re\left(\mu_{n}\right)\right|}\right)^{\frac{1}{2}}\left(\int_{-\frac{T}{2}}^{\frac{T}{2}}\left|\sum_{n \geq 1} a_{n} \mathrm{e}^{\mu_{n} t}\right|^{2} \mathrm{~d} t\right)^{\frac{1}{2}} .
\end{aligned}
$$

Hence, for any finite sequence $\left(a_{n}\right)_{n \geq 1}$, the following weighted Ingham type inequality holds

$$
\sum_{n \geq 1}\left|a_{n}\right|^{2} \mathrm{e}^{-2 \alpha\left|\Re\left(\mu_{n}\right)\right|} \leq C\left(T_{0}, a\right) \int_{-\frac{T}{2}}^{\frac{T}{2}}\left|\sum_{n \geq 1} a_{n} \mathrm{e}^{\mu_{n} t}\right|^{2} \mathrm{~d} t .
$$

\section{Controllability results}

This section is devoted to prove the main result of our paper.

Proof of Theorem 1.1. Let $T>\max \left\{2 \alpha, T_{0}+2 a\right\}$ and $\left(\zeta_{m}\right)_{m \in \mathbb{N}^{*}}$ as in Theorem 3.2. We construct a control $v_{\varepsilon} \in L^{2}(0, T)$ of $(1.5)$ corresponding to the initial datum $y^{0} \in H^{-1}(0, \pi)$,

$$
y^{0}(x)=\sum_{n \in \mathbb{N}^{*}} a_{n} \sin (n x),
$$

as follows:

$$
v_{\varepsilon}(t)=\sum_{n=1}^{\infty} \frac{(-1)^{n} \pi a_{n}}{2 n(\mathrm{i}+\varepsilon)} \mathrm{e}^{-\frac{T}{2} \bar{\mu}_{n}} \tilde{\zeta}_{n}\left(t-\frac{T}{2}\right) \quad \forall t \in(0, T),
$$

where $\tilde{\zeta}_{m}$ is the extension by zero of $\zeta_{m}$ to the interval $(-T / 2, T / 2)$. From the properties of the biorthogonal sequence $\left(\zeta_{m}\right)_{m \in \mathbb{N}^{*}}$, it is easy to see that $v_{\varepsilon}$ verifies $(2.2)$. To conclude that $v_{\varepsilon}$ is a control for (1.5), we only have to prove that the series from (4.2) converges in $L^{2}(0, T)$. This follows immediately from Theorem 3.2 and the fact that $y^{0} \in H^{-1}(0, \pi)$. Indeed, we have that

$$
\begin{aligned}
\int_{0}^{T}\left|v_{\varepsilon}(t)\right|^{2} \mathrm{~d} t & =\int_{0}^{T}\left|\sum_{n=1}^{\infty} \frac{(-1)^{n} \pi a_{n}}{2 n(\mathrm{i}+\varepsilon)} \mathrm{e}^{-\frac{T}{2} \bar{\mu}_{n}} \tilde{\zeta}_{n}(t-T / 2)\right|^{2} \mathrm{~d} t \\
& =\int_{-\frac{T_{0}}{2}-a}^{\frac{T_{0}}{2}+a}\left|\sum_{n=1}^{\infty} \frac{(-1)^{n} \pi a_{n}}{2 n(\mathrm{i}+\varepsilon)} \mathrm{e}^{-\frac{T}{2} \bar{\mu}_{n}} \zeta_{n}(t)\right|^{2} \mathrm{~d} t \\
& \leq C\left(T_{0}, a\right) \sum_{n=1}^{\infty} \frac{\pi^{2} a_{n}^{2}}{4 n^{2}} \frac{\mathrm{e}^{-T\left|\Re\left(\mu_{n}\right)\right|}}{1+\varepsilon^{2}} \mathrm{e}^{2 \alpha\left|\Re\left(\mu_{m}\right)\right|} \leq C\left(T_{0}, a\right) \frac{\pi^{2}}{4}\left\|y_{0}\right\|_{H^{-1}(0, \pi)}^{2} .
\end{aligned}
$$

Note that the constant from the last inequality $C\left(T_{0}, a\right)$ is the one from (3.24) and it does not depend of $\varepsilon$. Thus, the family of controls $\left(v_{\varepsilon}\right)_{\varepsilon>0}$ is uniformly bounded in $L^{2}(0, T)$. Let $v$ be a weak limit of this family. 
In order to show that $v$ is a control for equation (1.1) we only have to pass to the limit as $\varepsilon$ goes to zero in the characterization relation (2.2).

\section{Comments}

(1) Concerning the optimal controllability time. Theorem 1.1 ensures the existence of a time $T>0$, sufficiently large but independent of $\varepsilon$, for which the uniform controllability of (1.5) holds. As we have mentioned from the very beginning, the linear Schrödinger equation (1.1) is controllable in arbitrary small time. The same is probably true in our case. However, to obtain the optimal time we should be able to find a new product $P_{m}$ of arbitrarily small exponential type and a multiplier $M_{m, \varepsilon}$ with the constant $R$ from Lemma 3.3 as small as we want. This seems to be a difficult question and remains an open problem (see also $[12,25]$ for similar discussions).

(2) Concerning the product $P_{m}$. It is interesting to note that the simplest election for the function $P_{m}$ does not work. Indeed, if we choose

$$
P_{m}(z)=\prod_{\substack{n \in \mathbb{Z}^{*} \\|n| \neq m}}\left(1-\frac{z}{\mathrm{i} \bar{\mu}_{n}}\right)\left(\frac{\bar{\mu}_{n}}{\bar{\mu}_{n}-\bar{\mu}_{m}}\right),
$$

we have an entire function which verifies the necessary relations $P_{m}\left(\mathrm{i} \bar{\mu}_{n}\right)=\delta_{m n}$. Moreover, a product like (5.1), has arbitrarily small exponential type and eventually would allow to deduce a controllability result for any $T>0$.

However, $P_{m}$ given by $(5.1)$ is useless. Indeed, it is easy to see that

$$
\left|P_{m}(x)\right| \geq C_{1} \exp \left(C_{2} \sqrt{|x|}\right) \quad \forall x \in \mathbb{R}
$$

where $C_{1}$ and $C_{2}$ are positive constants independent of $\varepsilon$ and $m$. Consequently, for each $\varepsilon \in(0,1]$ and $m \geq 1$, we would need a multiplier $M_{m, \varepsilon}$ such that,

$$
\begin{gathered}
\left|M_{m, \varepsilon}(x)\right| \leq \exp \left(-C_{3} \sqrt{|x|}\right) \quad \forall x \in \mathbb{R}, \\
\left|M_{m, \varepsilon}\left(\mathrm{i} \bar{\mu}_{m}\right)\right| \geq \exp \left(-C_{4}\left|\Re\left(\mu_{m}\right)\right|\right)=\exp \left(-C_{4} \varepsilon m^{2}\right),
\end{gathered}
$$

with $C_{3}$ and $C_{4}$ two positive constants independent of $\varepsilon$.

We show that this is not possible. Indeed, suppose that, for each $\varepsilon \in(0,1]$ and $m \geq 1$, there exists an entire function $M_{m, \varepsilon}$ of exponential type less than $B>0$ and with properties (5.2), (5.3). If we take any $\eta>0$ and define the auxiliary function

$$
G_{m, \varepsilon}(z)=M_{m, \varepsilon}(z) \mathrm{e}^{(B+\eta) z} \mathrm{e}^{C_{3} \sqrt{-z}}
$$

we obtain from the Phragmén-Lindelöf Theorem that there exists a constant $C>0$, independent of $\varepsilon$ and $m$, such that

From the last inequality, we deduce that

$$
\left|G_{m, \varepsilon}(x+\mathrm{i} y)\right| \leq C, \quad x \leq 0, y \geq 0 .
$$

$$
\left|M_{m, \varepsilon}\left(\mathrm{i} \bar{\mu}_{m}\right)\right| \leq C \exp \left(-\frac{m}{\sqrt{2}}\right), \quad m \geq 1
$$

which contradicts (5.3). 
(3) Concerning rectangular domains. Our approach could be used to study the uniform controllability property of the perturbed two dimensional Schrödinger equation in a rectangular domain with a control located on an edge. In this case, the main problem is to deduce, for each $m \geq 1$, the existence of a bounded (in $m$ and $\varepsilon$ ) family of biorthogonal functions to $\left(\mathrm{e}^{\mu_{n m} t}\right)_{n \geq 1}$ when $\mu_{n m}=\varepsilon\left(n^{2}+m^{2}\right)-\mathrm{i}\left(n^{2}+\right.$ $\left.m^{2}\right)$. Although quite technical, this study could be carried out by using similar ideas as in [25].

(4) Concerning other spaces of controllable data and regular controls. In this article we have considered the space $H^{-1}(0, \pi)$ of controllable initial data which is the optimal space if one wants the controls to remain in $L^{2}(0, T)$. It is known that, if the initial data are more regular we can find smoother minimal $L^{2}$-norm boundary controls for the linear Schrödinger equation (see, for instance, [28]). The technique used in this paper may be adapted to study the controllability of more regular spaces of initial data (like $H^{s}(0, \pi), s \geq 0$ ) with smoother controls (like $H^{r}(0, T), r>0$ ). Indeed, more regular biorthogonal functions (and controls) may be constructed by considering in (3.21) Fourier transforms $\Psi_{m}$ such that $x^{r} \Psi_{m}(x) \in L^{2}(\mathbb{R})$ for some $r>0$. However, note that the corresponding controls are not of minimal $L^{2}$-norm.

(5) Concerning other controllability problems. The spectral approach we have used in this paper allows the study of other types of controls. Indeed, instead of looking for a boundary control $v_{\varepsilon}$ as in (1.5), we may be interested in finding an interior "lumped control" of the form $v_{\varepsilon}(t) g(x)$, where $g \in L^{2}(0, \pi)$ is a given function, or "punctual control" like $v_{\varepsilon}(t) \delta_{x_{0}}$, where $\delta_{x_{0}}$ is the Dirac mass concentrated in $x_{0} \in(0, \pi)$. It is well-known that the existence of such controls is equivalent to a moment problem similar to (2.2). The only major difference with respect to our study consists in deciding how the properties of the function $g$ or the position of the point $x_{0}$ affect the space of the controllable initial data in each of these cases.

(6) Concerning general domains and nonlinear equations. An interesting question is the possibility of extending our results to several dimension domains and even to nonlinear Schrödinger equation. The method from this paper, based on the explicit knowledge of the spectrum and the reduction to a linear moment problem, cannot be used any longer. A possibility would be to prove Carleman inequalities for the Ginzburg-Landau linear equation like the ones obtained in $[5,9-11,24,28]$, but independent of the parameter $\varepsilon$. This completely different technique will make the subject of a future work.

Acknowledgements. Both authors are partially supported by the bilateral project 19600SJ, Grant 206/2009 of ANCS (Romania). The first author is partially supported by Grant MTM2008-03541 funded by MICINN (Spain). The authors are grateful to Marius Tucsnak, Enrique Zuazua and Lionel Rosier for several interesting suggestions and comments.

\section{REFERENCES}

[1] O.M. Aamo, A. Smyshlyaev and M. Krstić, Boundary control of the linearized Ginzburg-Landau model of vortex shedding. SIAM J. Control Optim. 43 (2005) 1953-1971.

[2] S.A. Avdonin and S.A. Ivanov, Families of exponentials. The method of moments in controllability problems for distributed parameter systems. Cambridge University Press (1995).

[3] J. Ball and M. Slemrod, Nonharmonic Fourier series and the stabilization of distributed semi-linear control systems. Commun. Pure Appl. Math. XXXII (1979) 555-587.

[4] M. Bartuccelli, P. Constantin, C.R. Doering, J.D. Gibbon and M. Gisselfält, On the possibility of soft and hard turbulence in the complex Ginzburg Landau equation. Physica D 44 (1990) 421-444.

[5] L. Baudouin and J.-P. Puel, Uniqueness and stability in an inverse problem for the Schrödinger equation. Inverse Probl. 18 (2002) $1537-1554$.

[6] J.-M. Coron, Control and nonlinearity, Mathematical Surveys and Monographs 136. Am. Math. Soc., Providence (2007).

[7] J.-M. Coron and S. Guerrero, Singular optimal control: a linear 1-D parabolic-hyperbolic example. Asymptot. Anal. 44 (2005) $237-257$.

[8] R.J. DiPerna, Convergence of approximate solutions to conservation laws. Arch. Ration. Mech. Anal. 82 (1983) $27-70$.

[9] X. Fu, A weighted identity for partial differential operators of second order and its applications. C. R. Acad. Sci. Paris, Sér. I 342 (2006) 579-584.

[10] X. Fu, Null controllability for the parabolic equation with a complex principal part. J. Funct. Anal. 257 (2009) $1333-1354$. 
[11] A.V. Fursikov and O.Yu. Imanuvilov, Controllability of Evolution Equations, Lect. Notes Ser. 34. Seoul National University, Research Institute of Mathematics, Global Analysis Research Center, Seoul (1996).

[12] O. Glass, A complex-analytic approach to the problem of uniform controllability of a transport equation in the vanishing viscosity limit. J. Funct. Anal. 258 (2010) 852-868.

[13] L. Ignat and E. Zuazua, Dispersive Properties of Numerical Schemes for Nonlinear Schrödinger Equations, in Foundations of Computational Mathematics, Santander 2005, London Math. Soc. Lect. Notes 331, L.M. Pardo, A. Pinkus, E. Suli and M.J. Todd Eds., Cambridge University Press (2006) 181-207.

[14] L. Ignat and E. Zuazua, Numerical dispersive schemes for the nonlinear Schrödinger equation. SIAM J. Numer. Anal. 47 (2009) 1366-1390.

[15] A.E. Ingham, A note on Fourier transform. J. London Math. Soc. 9 (1934) 29-32.

[16] A.E. Ingham, Some trigonometric inequalities with applications to the theory of series. Math. Zeits. 41 (1936) $367-379$.

[17] J.P. Kahane, Pseudo-Périodicité et Séries de Fourier Lacunaires. Ann. Scient. Ec. Norm. Sup. 37 (1962) 93-95.

[18] V. Komornik and P. Loreti, Fourier Series in Control Theory. Springer-Verlag, New York (2005).

[19] M. Léautaud, Uniform controllability of scalar conservation laws in the vanishing viscosity limit. Preprint (2010).

[20] G. Lebeau, Contrôle de l'équation de Schrödinger. J. Math. Pures Appl. 71 (1992) 267-291.

[21] C.D. Levermore and M. Oliver, The complex Ginzburg Landau equation as a model problem, in Dynamical Systems and Probabilistic Methods in Partial Differential Equations, in Lect. Appl. Math. 31, Am. Math. Soc., Providence (1996) 141-190.

[22] A. López, X. Zhang and E. Zuazua, Null controllability of the heat equation as singular limit of the exact controllability of dissipative wave equations. J. Math. Pures Appl. 79 (2000) 741-808.

[23] E. Machtyngier, Exact controllability for the Schrödinger equation. SIAM J. Control Optim. 32 (1994) 24-34.

[24] A. Mercado, A. Osses and L. Rosier, Inverse problems for the Schrödinger equation via Carleman inequalities with degenerate weights. Inverse Probl. 24 (2008) 150-170.

[25] S. Micu and L. de Teresa, A spectral study of the boundary controllability of the linear 2-D wave equation in a rectangle. Asymptot. Anal. 66 (2010) 139-160.

[26] R.E.A.C. Paley and N. Wiener, Fourier Transforms in Complex Domains, AMS Colloq. Publ. 19. Am. Math. Soc., New York (1934).

[27] R.M. Redheffer, Completeness of sets of complex exponentials. Adv. Math. 24 (1977) 1-62.

[28] L. Rosier and B.-Y. Zhang, Null controllability of the complex Ginzburg Landau equation. Ann. Inst. Henri Poincaré, Anal. Non Linéaire 26 (2009) 649-673.

[29] M. Salerno, B.A. Malomed and V.V. Konotop, Shock wave dynamics in a discrete nonlinear Schrödinger equation with internal losses. Phys. Rev. 62 (2000) 8651-8656.

[30] M. Tucsnak and G. Weiss, Observation and Control for Operator Semigroups. Birkhäuser Advanced Texts, Springer, Basel (2009).

[31] R. Young, An Introduction to Nonharmonic Fourier Series. Academic Press, New York (1980).

[32] J. Zabczyk, Mathematical Control Theory: An Introduction. Birkhäuser, Basel (1992).

[33] X. Zhang, A remark on null exact controllability of the heat equation. SIAM J. Control Optim. 40 (2001) $39-53$. 